$\$=-$ 国

\title{
On cellular automata, traffic and dynamical systems in graphs
}

\author{
Buslaev Alexander P. ${ }^{1,2}$, Tatashev Alexander G. ${ }^{1,2}$, Yashina Marina V. ${ }^{1,2}$ \\ ${ }^{1}$ Moscow Automobile and Road Construction State Technical University (MADI), Moscow, 125319, Russian Federation \\ ${ }^{2}$ Moscow Technical University of Communications and Informatics (MTUCI), Moscow, 111024, Russian Federation \\ *Corresponding author E-mail: apal2006@yandex.ru
}

\begin{abstract}
Qualitative studies of discrete dynamical systems behavior on networks are relevant in many fields such as system biology, transportation, information traffic, material sciences and so on. We consider cellular automata on one-dimensional and two-dimensional toroidal supporters. At every discrete time moment, each cell of a cellular automaton is in one of two states 0 and 1 . We introduce concept of the cellular automaton mass at fixed time. The cellular automaton mass is the quantity of cells such that these cells are in the state 1 . The mass conservation law takes place if the mass of cellular automaton is the same at every time. Concepts of explosion and annihilation have been introduced. Explosion takes place if the mass of cellular automaton increases at each iteration until all cells are in the state 1. Annihilation takes place if the mass of cellular automaton decreases at each iteration until all cells are in the state 0 . We consider classes of cellular automata such that the state of cell at the next time depends on the state at current time and states of neighboring cells belonging to fixed set. We have found sets of cellular automata such that the mass conservation law, explosion or annihilation takes place for these automata.
\end{abstract}

Keywords: Dynamical Systems; Cellular Automaton; Wolfram Automata; Regular Networks; Traffic Flow.

\section{Introduction}

\subsection{The cellular automaton concept}

The concept of cellular automaton has been introduced by S. Ulam and J. von Neumann, ([1], 1963, [2], 1966, [3], 1974) for the modeling of biological systems. Such biological processes as reproduction and the evolution of organized forms can be modeled by cells that obey simple rules. The cellular automaton is onedimensional or multidimensional lattice, each cell of which at any time is in one of the states. Machine state in the next step is determined by a given rule and depends on from the state of this cell and the cells closest to it at the current moment.

J.H. Conway in 1970 introduced the cellular automaton "Life", [4]. In this cellular automaton, cells form a two-dimensional structure, the next state depends on the current state this cell and eight neighboring ones. Currently many examples of the behavior of the system are known for given initial states, but little analytical work on the general behavior of the given cellular automaton. In [5], with reference on the monograph of Z. Manna ([6], 1974), it is noted that Conway's cellular automaton on an infinite cellular field is a computing device for the formalization of the work of algorithms.

\subsection{Wolfram's cellular automata}

S.Wolfram, [5], 1982, has introduced the class of elementary cellular automata. Cells of these automata are located on infinite one-dimensional lattice and are numerated successively. Every cell is in one of two states 0 and 1 at each discrete moment $t=0,1,2, \ldots$ The cell state at time $t+1$ is determined fully by the state of this cell and neighboring cells at time $t$. The state is formalized by the following map of binary variables
There exist 256 cellular automata. Numbers from 0 to 255 are assigned to these automata in accordance with rules described in Section 4. A set of cellular automata called legal automata is introduced in [5]. The set of legal automata satisfies the following conditions. The initial null configuration will be ever null configuration. This is fulfilled if and only if $\operatorname{CA}(0,0,0)=0$. Moreover, the automaton is symmetrical, i. e.,

$\mathrm{CA}\left(\mathrm{x}_{\mathrm{i}-1}(\mathrm{t}), \mathrm{x}_{\mathrm{i}}(\mathrm{t}), \mathrm{x}_{\mathrm{i}+1}(\mathrm{t})\right) \equiv \mathrm{CA}\left(\mathrm{x}_{\mathrm{i}+1}(\mathrm{t}), \mathrm{x}_{\mathrm{i}}(\mathrm{t}), \mathrm{x}_{\mathrm{i}-1}(\mathrm{t})\right)$.

In [7], (S. Wolfram, 1984), the classification of [5] is generalized for cellular automata such that each cell of a automata is in one of $\mathrm{k} \geq 2$ states, $0, \ldots, \mathrm{k}-1$, and the state of $\mathrm{i}$-th cell at time $\mathrm{t}+1$ depends on the state of this cell at time $t$, states of $r$ cells with indexes $i-r, \ldots, i-1$ and states of $r$ cells with indexes $i+$ $1, \ldots, i+r$. In accordance with numeration of the work [7], which is similar to numeration of [5], all considered automaton have numbers from 0 to $\left(\mathrm{k}^{\mathrm{k}^{2 \mathrm{r}+1}}-1\right)$, where $\mathrm{k}$ and $\mathrm{r}$ are given. In Wolfram's papers, it has been described the possible applications of cellular automata for formal description of algorithms and formal grammars, or for the study of entropy in theoretical mechanics models, etc., [5]-[9].

We note that in [6], it is assumed that, in initial moment only finite quantity of cells on infinite fields are in the state 1. In [6]-[8] it is indicated that there exist following forms of cellular automata behavior:

a) From a time moment, all cells are in the state 1 .

b) From a time moment, the quantity of cells, being in state 1 , does not change.

c) The quantity being in state 1 , increases unboundedly.

d) Time intervals alternate. They are time intervals of occupied cells increasing and occupied cells decreasing. 
In [9], O. Martin, A.M. Odlyzko, S. Wolfram, 1984, algebraic approaches and approaches of the number theory are used for analysis and investigation of cellular automata properties.

In [10], a class of cellular automata, called additive, is mainly considered. These cellular automata satisfy superposition principle. This principle allows represent configurations by characteristic polynomials. Evolution of configurations is represented be iterative multiplication of characteristic polynomials by a fixed polynomial. General properties of cellular automata are determined by algebraic properties of these polynomials.

It is assumed in [10] that cellular automaton contains $\mathrm{N}$ cells located on a circle. States (values) of cells at time $t$ are denoted by $a_{0}^{(t)}, \ldots, a_{N-1}^{(t)}$. In general case, numbers of cells are elements of $a$ commutative ring. The number of these elements equals $\mathrm{k}$. In the simplest case, cells can be in two states 0 and 1 . The configuration of the cellular automaton is determined by states of $\mathrm{N}$ cells and can be represented by characteristic polynomial (generating function) $A^{(t)}(x)=\sum_{i=0}^{N-1} a_{i}^{(t)} x^{i}$, where the value of the cell $i$ is the coefficient of $x_{i}$. Configuration is identified with characteristic polynomial. In [10], a special class of additive cellular automata is considered. These automata function in accordance with a simple linear combination of the following rules (addition and subtraction modulo $\mathrm{N}$ in indexes)

$\mathrm{a}_{\mathrm{i}}^{(\mathrm{t})}=\alpha_{-1} \mathrm{a}_{\mathrm{i}-1}^{(\mathrm{t}-1)}+\alpha_{0} \mathrm{a}_{\mathrm{i}}^{(\mathrm{t}-1)}+\alpha_{+1} \mathrm{a}_{\mathrm{i}+1}^{(\mathrm{t}-1)}$

Time evolution is represented by multiplication of the characteristic polynomial by the fixed dipolyninomial

$\mathrm{T}(\mathrm{x})=\alpha_{-1} \mathrm{x}+\alpha_{0}+\alpha_{+1} \mathrm{x}^{-1}:$

$A^{(t)} \equiv T(x) A^{t-1}(x) \bmod \left(x^{N}-1\right)$.

The cellular automaton submits to additive principle of superposition. In accordance with this principle, the configuration $\mathrm{A}^{(\mathrm{t})}(\mathrm{x})+\mathrm{B}^{(\mathrm{t})}(\mathrm{x})$ is the result of automaton functioning for initial configuration $\mathrm{A}^{(0)}(\mathrm{x})+\mathrm{B}^{(0)}(\mathrm{x})$ if $\mathrm{A}^{(\mathrm{t})}(\mathrm{x}), \mathrm{B}^{(\mathrm{t})}(\mathrm{x})$ are results of configurations $A^{(0)}(x)$ and $B^{(0)}(x)$ evolutions. Therefore any initial configuration can be represented as a sum of basis configurations $\mathrm{A}(\mathrm{x}) \equiv \mathrm{x}^{\mathrm{j}}$ containing a single cell with non-zero state, and the additive principle determines the evolution of configurations in terms of evolution of $(x)$. Classical facts of number theory are used in proving a theorem which gives the period with which states of additive cellular automaton are returned.

In [10], N.H. Packard, S. Wolfram, 1985, cellular automata were considered on two-dimensional lattice. As important particular cases, automata are considered such that, in these automata, the next state of cell is determined by state of this cell at current moment and states of 4 neighboring cells (neighborhood of von Neumann) or determined by state of this cell at current moment and states of 8 neighboring cells (it neighborhood of Moore). Conway cellular automaton is a particular case of the automaton with neighborhood of Moore. Results and analysis of simulation are given for fixed initial states. The concept of entropy of is used for study of automata behavior.

\subsection{Cellular automata are traffic models}

In 1992, a two-dimensional traffic model has been introduced, [11], Biham O., Middleton D., Levin D. In these model, cells form a twodimensional toroidal structure. At every moment, each cell in one of two states 0 or 1 . We assume that a cell is occupied by a particle if this cell is in the state 1 , and the cell is vacant if it is in the state 0 . There are particles of two types. They are red particles and blue particles. Red particles move only to the right, and blue particles move only upwards. Moments such that only red particles move and moments such that only blue particles move alternate. In each direction, particles move in accordance with rule CA 184 Simulation shows that increase of flow density results in jump from it self-organization (from any initial state, after a finite time, the system comes to the state such that all particles move without delays) to it collapse (motion ceases).

In 2005, [17], R.M. D'Souza, it is found that, for some densities of traffic in BML model, there exists an intermediate phase. This phase is characterized by periodic configurations such that jams and free flow alternate. O. Angel, A.E. Holroyd, J.B. Martin, [18], have proved that jam ever is formed in BML model if the flow density is close to 1. In 2006, T. Austin and I. Benjamini, [19], have proved that self-organization takes place if the number of particles is less than $\frac{N}{2}$. Besides other systems, in monograph [20], Kozlov V.V.,Buslaev A.P., Tatashev A.G.,2013, the system with a supporter, dual to BML, was considered. The difference is also that there is only one type of particles and moments such that all particles move to the right and moments such that all particles move upwards alternate. Moreover it is assumed that, if movement of a particle is allowed, then the particle moves with a fixed probability, and, in general case, this probability depends on direction of movement. A formula has been obtained for the velocity in the fixed direction provided that the probability of movement at each moment is small regarding at least one of coordinates. In [15], Kozlov, Buslaev, Tatashev, 2015, and [21], Buslaev, Tatashev, 2018 , class of dynamical systems was considered such that, in these system, supporters are systems of contours, and particles move on cells of these contours. Each particle moves on its contour. Particles interact with each other in common points called nodes. On contours, particles move in accordance with rules CA 184 or 240. In the case of rule 240, particles of each cluster move synchronously. In [22], Kozlov, Buslaev, Tatashev, 2015, and [23], 2016, Buslaev, Tatashev, dynamical systems are introduced such that, in these systems, movement of particles on cells is determined by plans. Plans are sequences of symbols. These sequences can be represented on a moving tape. We call this tape Turing tape. In particular case, when there are two cells and two particles (bipendulum) there is a sequence of zeros and ones on the tape. This sequence is the binary representation of the fractional part of a rational number belonging to the segment moving $[0,1]$.

We assume that, at every moment, each particle reads the first symbol on its tape. At next moment, the tape shifts to one position to the left. The symbol read by the particle is erased, and other symbols shift one position to the left. Delay in the implementation of the particles plans are due to particles interacting. If we consider the binary representation on the tape as a cellular automaton, then this representation change in accordance with cellular automaton 170, which corresponds to movement similar to movement described by CA 240 but particles move in the opposite direction. In cellular automaton 240 , the next state of each cell depends only on the state of this cell at the current moment and the state of the neighboring cell on the left. Similarly, in cellular automaton 170 , the next state of each cell depends only on the state of this cell at the current moment and the state of the neighboring cell on the right. Therefore there are no difficulties in descriptions of movement related to that the tape is bounded on the left. An equivalent approach to describe the tape movement is to apply the map called the Bernoulli shift. If the Bernoulli shift is applied to a number belonging to the segment $[0,1]$, then this number is multiplied by 2 and the integer part of the number is excluded. Unary algebras on the set of common fractions with the operation of Bernoulli shift were considered in [22], [24], (Buslaev, Tatashev, 2016). Generalizations of the bipendulum (transport and logistics problem) and Bernoulli algebras were considered in [23], [25]. These generalizations correspond to generalization of CA170 to the case of more than 2 possible states of each cell (cells of the cellular automaton correspond to binary digits on the tape).

\subsection{Formulation of problem}

\subsubsection{Architecture of CA}

Let us describe classes of cellular automata considered in this paper. In general case, we consider multi-dimensional structures. These structures can be open, infinite in one direction, infinite in two 
directions, or toroidal. In one-dimensional case, we consider a ring or periodic sequences of cells, and these sequences are infinite on one or two directions. The next state of each cell is determined by the current state of this cell and states of a fixed quantity $r$ or 1 of cells on the right and on the left correspondingly.

\subsubsection{Cellular automata on a circle}

We consider the following class of one-dimensional cellular automaton. Integer nonnegative numbers $r, 1$ are given. The state of each cell can be equal to 0 or 1 , and the state of the cell $i$ at time $t+1$ is determined by the state at time $t$ of this cell and cells $\mathrm{i}-\mathrm{s}, \ldots, \mathrm{i}-$ $1, i, i+1, \ldots, i+r$.

In these terms, we have $\mathrm{r}=\mathrm{l}=1$ for the class of Wolfram elementary automata.

\subsubsection{Cellular automata on a torus}

We consider also the following classes of two-dimensional cellular automata.

N) Integer nonnegative numbers $r_{1}, l_{1}, r_{2}, l_{2}$ are given. The state of any cell takes two possible values 0,1 , and the state of cell $\left(i_{1}, i_{2}\right)$ at moment $t+1$ is determined by the state of this cell at time $t$ and cells $\left(\mathrm{j}_{1}, \mathrm{j}_{2}\right)$ such that

$\mathrm{i}_{\mathrm{k}}-\mathrm{l}_{\mathrm{k}} \leq \mathrm{j}_{\mathrm{k}} \leq \mathrm{i}_{\mathrm{k}}+\mathrm{r}_{\mathrm{k}}, \mathrm{k}=1,2$.

W) A integer nonnegative number $r$ is given. The state of the cell $\left(i_{1}, i_{2}\right)$ at moment $t+1$ is determined by the state of this cell at moment $t$ and states of cells $\left(\mathrm{j}_{1}, \mathrm{j}_{2}\right)$ such that

$\max \left|j_{k}-i_{k}\right| \leq r$.

In these terms, we have $r=1$ for the class of one-dimensional elementary automata of Wolfram and for the class of twodimensional automata of Conway.

In Section 5 we consider two-dimensional automaton such that for this automaton $r_{1}=r_{2}=0, l_{1}=l_{2}=1$, i.e., for this automaton, the state of the cell $(i, j)$ at time $t+1$ depends on the state of cells $(i, j),(i-1, j)$ and $(i, j-1)$, at time $t$.

\subsubsection{Characteristics and conservation laws}

We shall give the following definitions. The quantity of cells being in state 1 at current moment is called the mass of the cellular automaton. The property of system to conserve its mass is called mass conservation law. We consider the problem to find all cellular automata of considered class such that the conservation law is fulfilled for these automata. We formulate also the problem to find cellular automata such that their mass conserves from a moment For infinite automata with non-periodic structure, we define the concept of density of the cellular automaton and formulate the problem to find automata such that, for these automata, its density conserves at each iteration, or the density conserves after a moment, or the density tends to a fixed value. The following problems are interesting. They are the problem to find cellular automata of a given class such that, in fixed cluster, the quantity of ones satisfy conditions for the mass. For example, this quantity increases to maximum (explosion) or decreases to zero (annihilation) for finite time.

\section{Cellular automata of minimum dimension $\mathbf{w}_{1}$}

Assume that $r=1=0$, i. e., in the case of any dimension of CA structure, the state of the cell depends on only the state of this state at the precedent moment. There are 4 cellular automaton which are determined by the following maps $0 \rightarrow 0,1 \rightarrow 0$ (cellular automaton 0 ), $0 \rightarrow 0,1 \rightarrow 1$ (cellular automaton 1), $0 \rightarrow 1,1 \rightarrow 0$ (cellular automaton 2), $0 \rightarrow 1,1 \rightarrow 1$ (cellular automaton 3 ). Only automaton 1 conserves mass. The automaton 0 corresponds to annihilation, and automaton 1 corresponds the explosion.

\section{Wolfram cellular automata $\mathbf{w}_{2}$}

In Section 3 we consider two classes of a simpler automaton such that the value of digits depends on state only one neighboring digit (on state of the digit ahead).

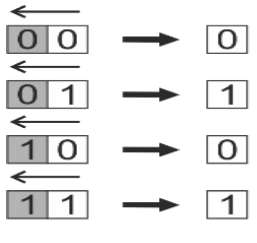

a)

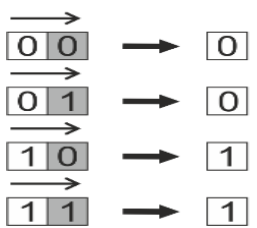

c)

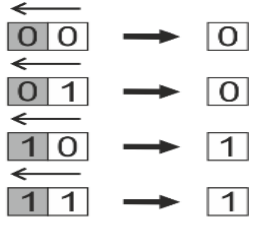

b)

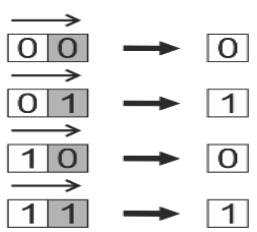

d)
Fig. 1: Cellular Automata Conserving Mass.

Consider automaton defined by Table 3.1. In accordance with this table, digit 0 is replaced by $c_{0}$ if, in the current state, the next digit is also 0 , and the digit is replaced by $c_{1}$ if the next digit is 1 . Digit 1 is replaced by $c_{2}$ if, in the current state, the next digit is 0 , and the digit is replaced by $c_{3}$ if the next digit is 1 .

Table 3.1: Cellular Automaton $W_{2}$

\begin{tabular}{lcccc}
\multicolumn{6}{c}{ Table 3.1: Cellular Automaton $W_{2}$} \\
\hline 00 & 01 & 10 & 11 & \\
\hline$c_{0}$ & $c_{1}$ & $c_{2}$ & & $c_{3}$ \\
\hline
\end{tabular}

The number with binary representation $c_{3} c_{2} c_{1} c_{0}$ is assigned to the cellular automaton determined by Table 3.1. The considered class contains 16 cellular automata.

Proposition 3.1 (mass conservation criterion). In considered class there are just 2 cellular automata 10 and 12 conserving mass. Cellular automaton 12 corresponds to total-connected movement in direction of increase of coordinate, and cellular automaton 30 determines the identity map.

Proof. Since the map conserves periodic chains (0), (1), we have

$c_{0}=0, c_{3}=1$.

We consider the change of periodic chain (01). We have the equation

$c_{1}+c_{2}=1$

Taking into account (4) we have that either $c_{1}=0, c_{2}=1$ or $c_{1}=$ $1, c_{2}=0$. In the considered case, there are only two automata $2^{3}+$ $2=10,2^{3}+2^{2}=12$ such that these automata satisfy conditions (3), (4). It is easy to prove that the automata 10 and 12 conserve the mass.

Proposition 3.2 (criterion of annihilation). A necessary and sufficient condition for the mass to decrease at any iteration is that the rule 0 be fulfilled (0-map).

Proof. Considering maps of periodic configurations, considered in proof of Proposition 2.1, we get that for mass to decrease is necessary to have $c_{0}, c_{1}+c_{2}<1, c_{3}<1$. From this we have Proposition 3.2.

Proposition 3.3 (criterion of explosion). A necessary and sufficient condition for the mass to increase at any iteration is that the rule 15 be fulfilled (1-map). 
The proof of Proposition 3.3 is similar to proof of Proposition 3.1. Proposition 3.4. (criterion of mass monotony) a) A necessary and sufficient condition for the mass not to increase at any iteration is that one of the rules $0,2,4,8,10,12$ be fulfilled. b) A necessary and sufficient condition for the mass not to decrease at any iteration is that one of the rules $10,11,12,13,14,15$ be fulfilled.

Proof. a) Considering maps of periodic configurations, considered in proof of Proposition 3.1, we get that for mass to decrease it is necessary to have $c_{0}, c_{1}+c_{2} \leq 1$. Automata $0,2,4,8,10,12$ satisfy these conditions. Taking into account that, on a period, the quantity of pairs 01 is equal to the quantity of pairs 10 , we get that these automata satisfy the condition that the mass does not increase. b) Consider the class of automata determined by a table, similar to Table 3.1, but such that, for this automaton, the value of each cell at the next moment depends on current state of this digit and the neighboring digit on the right. For this class of automata, statements, similar to Propositions 3.1-3.4, but now cellular automaton 12 corresponds to the identical map and cellular automaton 10 corresponds to the total-connected movement in the direction of coordinate decrease.

\section{Cellular automata $w_{3}$}

\subsection{Description of state of cellular automaton $3 \times 1$}

Consider the set of rational numbers $Q \cap[0,+\infty)$ in binary representation in the follow form

$x=0 . a_{1} \ldots a_{m}\left(b_{1} \ldots b_{n}\right)$,

Where $a_{i}, b_{j} \in 0,1$. Suppose $[a]$ is the integer part of real number, $a-[a]$ is the fractional part, $B x=2 x-[2 x]$ is the Bernoulli map. Finite sequence $x_{1}=0 .\left(b_{1} \ldots b_{n}\right), x_{2}=0 .\left(b_{2} \ldots b_{n} b_{1}\right), \ldots, x_{n}=$ $0 .\left(b_{n} b_{1}, \ldots, b_{n-1}\right)$ is called orbit of the number (5) and all numbers $x_{i}$ are different. It is obvious that $B\left(x_{1}\right)=x_{2}, B\left(x_{2}\right)=$ $x_{3}, \ldots, B\left(x_{4}\right)=x_{1}$ and we have a simple cycle of rational numbers To each row $\left(b_{n}, b_{1}, b_{2}\right),\left(b_{1}, b_{2}, b_{3}\right), \ldots,\left(b_{n-1}, b_{n}, b_{1}\right)$ assign numbers $c_{1}, c_{2}, \ldots, c_{n} \in\{0,1\}$ and $\left.y_{1}=0 .\left(c_{1} \ldots c_{n}\right) \ldots c_{n}\right)$. Numbers $y_{1}, y_{2}=B\left(y_{1}\right), y_{3}=B\left(y_{2}\right), \ldots, y_{1}=B\left(y_{n}\right)$ form a cycle (this cycle can be not simple).

\subsection{Classification of $3 \times 1$ automata}

Define the following automaton by Table 4.1.

Table 4.1: Definition of $\mathrm{Ca} 184$

\begin{tabular}{llllllll}
\hline 000 & 001 & 010 & 011 & 100 & 101 & 110 & 111 \\
\hline 0 & 0 & 0 & 1 & 1 & 1 & 0 & 1 \\
\hline
\end{tabular}

$10111000_{2}=2^{7}+2^{5}+2^{4}+2^{3}=128+32+16+8=184$

We shall give another example.

Table 4.2: Definition of CA 240

\begin{tabular}{llllllll}
\hline 000 & 001 & 010 & 011 & 100 & 101 & 110 & 111 \\
\hline 0 & 0 & 0 & 0 & 1 & 1 & 1 & 1 \\
\hline
\end{tabular}

$11110000_{2}=2^{7}+2^{6}+2^{5}+2^{4}=128+64+32+16=240$

\subsection{Turing dial}

Let us define graph of threes of sequent digits processing in accordance with given automaton $W_{3}=W_{3 \times 1}$ Each cyclic sequence $\left(b_{1} \ldots b_{n}\right)$ is processed as movement on a graph. A closed path on a graph such that this path enters anyvertex no more than one time is called a simple cycle.

\subsection{Mass conservation law}

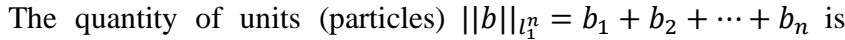
called the mass of the sequence $\left(b_{1} \ldots b_{n}=b\right.$. The principle problem is to describe class of $3 \times 1$ automata $\{0,1, \ldots, 255\}$ such that these automata conserves the mass, i. e., for any cyclic vector $\left(b_{1} \ldots b_{n}\right.$, we have

$x=0 . a_{1} \ldots a_{m}\left(b_{1} \ldots b_{n}\right)$,

Proposition 4.1. Mass conservation law is fulfilled if and only if it is fulfilled for any simple cycle.

The proof is evident.

Theorem 4.2: Mass conservation law for automata $3 \times 1$ is fulfilled only for cellular automata $\Leftrightarrow 170,184,204,226,240$.

Proof. Let us enumerate all simple cycles.

Cycles of length 1 .

$c_{0}=0 \Leftrightarrow x=0$. (0),

$c_{7}=1 \Leftrightarrow x=0 .(1)$.

Cycles of length 2 .

$c_{2}+c_{5}=1 \Leftrightarrow x=0 .(01) \sim 0 .(10)$.

Cycles of length 3 .

$c_{1}+c_{2}+c_{4}=1 \Leftrightarrow x=0 .(001) \sim 0 .(010) \sim 0 .(100)$

$c_{3}+c_{6}+c_{5}=2 \Leftrightarrow x=0 .(011) \sim 0 .(110) \sim 0 .(101)$

Cycles of length 4 .

$c_{0}+c_{4}+c_{2}+c_{4}=1 \Leftrightarrow 0 .(0001)$

$c_{3}+c_{7}+c_{6}+c_{5}=2 \Leftrightarrow 0 .(0111)$

$c_{1}+c_{3}+c_{6}+c_{4}=2 \Leftrightarrow 0 .(0011)$

Cycles of length 5 .

$c_{0}+c_{1}+c_{3}+c_{6}+c_{4}=2 \Leftrightarrow 0 .(00011)$

Cycles of length 6 .

$c_{0}+c_{1}+c_{3}+c_{7}+c_{6}=3 \Leftrightarrow 0 .(000111)$

It follows from (11) that for mass conservation law to be fulfilled it is necessary to have

$c_{2}=1, c_{5}=0$, or

$c_{2}=0, c_{5}=1$.

From (18), taking into account (12), (13), we get

$c_{1}+c_{4}=0$,

$c_{3}+c_{6}=2$.

From (18), (19), we get

$c_{1}=c_{4}=0, c_{3}=c_{6}=1$.

From (9), (10), (11), (12), we get that the cellular automaton, for that

$c_{0}=c_{1}=0, c_{2}=c_{3}=1, c_{4}=c_{5}=0, c_{6}=c_{7}=1$,

i) e., the cellular automaton 
$2^{7}+2^{6}+2^{3}+2^{2}=204$.

If (19) is fulfilled, then taking into account (12), (13) we get

$c_{1}+c_{4}=1$

$c_{3}+c_{6}=1$.

From (21), (22) we get that

$$
\begin{aligned}
& c_{1}=0, c_{4}=1, c_{3}=0, c_{6}=1, \text { or } \\
& c_{1}=0, c_{4}=1, c_{3}=1, c_{6}=0, \text { or } \\
& c_{1}=1, c_{4}=0, c_{3}=0, c_{6}=1, \text { or } \\
& c_{1}=1, c_{4}=0, c_{3}=1, c_{6}=0 .
\end{aligned}
$$

From (9), (10), (15), (21), we get

$c_{0}=c_{1}=c_{2}=c_{3}=0, c_{4}=c_{5}=c_{6}=c_{7}=1$,

and therefore cellular automaton $2^{7}+2^{6}+2^{5}+2^{4}=240$ conserves the mass.

From (5), (6), (15), (22), we have

$c_{0}=c_{1}=c_{2}=0, c_{3}=1, c_{4}=c_{5}=1, c_{6}=0, c_{7}=1$,

And therefore the cellular automaton $27+25+24+23=184$ conserves the mass.

From (5), (6), (15), (23), we get

$c_{0}=0, c_{1}=1, c_{2}=0, c_{3}=1$,

$c_{4}=0, c_{5}=1, c_{6}=0, c_{7}=1$,

And therefore cellular automaton $2^{7}+2^{6}+2^{5}+2=226$ conserves the mass.

From (5), (6), (15), (24), we get

$c_{0}=0, c_{1}=1, c_{2}=0, c_{3}=0$,

$c_{4}=0, c_{5}=1, c_{6}=1, c_{7}=1$,

And therefore cellular automaton $2^{7}+2^{6}+2^{5}+2=170$ conserves the mass.

Theorem 4.2 has been proved.

Remark 4.1: Mechanical sense of obtained rules is the following.

1) CA 184 is an individual motion of particles on a contour of cells in the same direction (clockwise), [12] .

2) CA 226 is individual motion counterclockwise.

3) CA 240 is total-connection motion clockwise, [22].

4) CA 170 is total-connection motion counterclockwise.

5) CA 204 is identity map.

\subsection{Annihilation and explosion}

Proposition 4.3 (annihilation criterion). A necessary and sufficient condition for the mass to decrease at any iteration of Turing dial it that one of the rules 008, 064 or the O-map.

Proof. For the mass to decrease it is necessary to have

$c_{0}=0$,

$c_{7}<1 \Leftrightarrow c_{7}=0$,

$c_{2}+c_{5}<1 \Leftrightarrow c_{2}=c_{5}=0$, $c_{1}+c_{2}+c_{4}<1 \Leftrightarrow c_{1}=c_{4}=0$

$c_{3}+c_{6}+c_{5}<2 \Leftrightarrow c_{3}+c_{6} \leq 1, \Rightarrow\left(c_{3}=0, c_{6}=1,\right) \vee\left(c_{3}=\right.$

$\left.1, c_{6}=0\right)$,

$c_{1}+c_{3}+c_{6}+c_{4}<2$,

$c_{1}+c_{3}+c_{6}+c_{4}<3$.

In accordance with (25)-(31) we have

$c_{0}=c_{1}=c_{2}=c_{3}=c_{4}=c_{5}=0, c_{6}=1, c_{7}=0$, or

$c_{0}=c_{1}=c_{2}=0, c_{3}=1, c_{4}=c_{5}=c_{6}=c_{7}=0$.

Therefore the annihilation corresponds to rules 0,008 or 064 .

Similarly we obtain the following.

Proposition 4.4 (explosion criterion).A necessary and sufficient condition for the mass to increase at any iteration of Turing dial is that one of the rules 247,253 or the 1-map.

\section{Simplest two-dimensional automata}

Consider a two-dimensional generalization of the simplest cellular automaton. Assume that a cellular automaton is defined on the toroidal system of cells $(i, j), i=0,1, \ldots, m, j=0,1, \ldots, n,(n \geq 3$, $m \geq 3$ ). Each of these cells is in state 0 or 1 at every moment $T=$ $0,1,2, \ldots$ The automaton is defined by a table, similar to Table 3.1, but now, if in $k$-th column of the table, row $b_{1} b_{2} b_{3}$ is given above and the digit $c_{k}$ below, then this means the following. If the cell $(i, j)$ is in the state $b_{2}$, the cell $(i-1, j)$ (substraction modulo $m$ ) is in the state $b_{1}$, and the cell $(i-1, j)$ (substraction modulo $\left.n\right)$ is in the state $b_{3}$, then, at the next moment, the cell $(i, j)$ is in the state $c_{k}, k=0, \ldots, 7$.

This class contains 256 cellular automata. The numeration of these automata is analogous to the numeration of cellular automata considered in Sections 2 - 4.

Cellular automata, conserving the mass, are defined such as for onedimensional case.

Theorem 5.1. In considered class, there are just 3 cellular automata conserving the mass. They are automata 170, 204, 240.

Proof. Since the map conserves the matrix, containing only zeros, and the matrix, containing only ones,

$c_{0}=0, c_{7}=1$.

Suppose we have matrix, got by repeating the matrix

$\left(\begin{array}{ll}0 & 0 \\ 0 & 1\end{array}\right)$

In the direction of both coordinate axes. Considering the map of this matrix, we get

$c_{0}+c_{1}+c_{2}+c_{4}=1$.

From (32), (33), we get

$c_{1}+c_{2}+c_{4}=1$

Suppose we have matrix having got by repeating the matrix

$\left(\begin{array}{ll}0 & 0 \\ 1 & 1\end{array}\right)$

In the direction of both coordinate axes. Considering the map of the matrix, we get

$c_{1}+c_{6}=1$.

Similarly, considering matrices 
$\left(\begin{array}{ll}0 & 1 \\ 0 & 1\end{array}\right)$

$\left(\begin{array}{ll}1 & 0 \\ 0 & 1\end{array}\right)$

We get equations

$c_{3}+c_{4}=1$

$c_{2}+c_{5}=1$.

If $c_{2}=1$ then, from (32), (33)-(37) we get

$c_{0}=0, c_{1}=0, c_{2}=1, c_{3}=1$,

$c_{4}=0, c_{5}=0, c_{6}=1, c_{7}=1$,

i. e., we have cellular automaton 204.

Similarly, assuming that $C_{4}=1$ we get

$c_{0}=0, c_{1}=0, c_{2}=0, c_{3}=0$,

$c_{4}=1, c_{5}=1, c_{6}=1, c_{7}=1$,

This corresponds to cellular automaton 240 .

Assuming that $c_{2}=1$ we get

$c_{0}=0, c_{1}=1, c_{2}=0, c_{3}=1$,

$c_{4}=0, c_{5}=1, c_{6}=0, c_{7}=1$,

i. e., we have cellular automaton 170 . Thus,

$c_{0}=0, c_{1}=0, c_{2}=1, c_{3}=1$,

$c_{4}=0, c_{5}=0, c_{6}=1, c_{7}=1$,

$c_{0}=0, c_{1}=0, c_{2}=0, c_{3}=0$,

$c_{4}=1, c_{5}=1, c_{6}=1, c_{7}=1$,

$c_{0}=0, c_{1}=1, c_{2}=0, c_{3}=1$,

$\mathrm{c}_{4}=0, \mathrm{c}_{5}=1, \mathrm{c}_{6}=0, \mathrm{c}_{7}=1$

It is easy to prove that cellular automata 170, 204, 240 conserve the mass.

\section{Theorem 5.1: Has been proved.}

Remark 5.1: Cellular automaton 204 corresponds to the identity map. Cellular automaton 240 corresponds to total-connected movement in the direction of the first coordinate increase. Cellular automaton 170 corresponds to total-connected movement in the direction of the second coordinate increase.

Proposition 5.2 (annihilation criterion). A necessary and sufficient condition for the mass to decrease at any iteration it that the rule 0 be fulfilled (0-map). Proof. Considering maps of matrices, considered in the prove of Theorem 5.1, we get

$\mathrm{c}_{0}=0, \mathrm{c}_{7}<1$,

$\mathrm{c}_{1}+\mathrm{c}_{2}+\mathrm{c}_{4}<1$,

$\mathrm{c}_{1}+\mathrm{c}_{6}<1$

$\mathrm{c}_{3}+\mathrm{c}_{4}<1$,

$\mathrm{c}_{2}+\mathrm{c}_{5}<1$.

From this Proposition 5.2 follows.

Proposition 5.3 (explosion criterion). A necessary and sufficient condition for the mass to decrease at any iteration it that the rule
255 be fulfilled (1-map). The proof of Proposition 5.3 is similar to the proof of Proposition 5.2.

\section{Acknowledgements}

This work has been supported by the Russian Foundation for Basic Research, Grant No. 17-01-00821 and Grant No. 17-07-001358.

\section{References}

[1] Von Neumann J. 1963. The general and logical theory of automata In J. von Neumann, Collected works, edited by A.H. Taub, 288.

[2] Von Neumann J. 1966. Theory of self-reproducing automata, edited by A.W. Burks (University of Illions, Urnana).

[3] Ulam S. Some ideas and prospects in bio-mathematics. Ann.Rev.Bio. 255 (1974).

[4] Berlekamp E.R., Conway J.H., Gay R.K. Winning ways for your mathematical plays. A.K. Peters Ltd, 2001-2004. 4 vols.

[5] Wolfram S. Cellular Automata as Simple Self-Organizing Systems. California Institute of Technology, Pasadena CA 91125 (July 1982; revised November 1982)

[6] Manna Z. Mathematical Theory of Computation, McGraw-Hill, 1974.

[7] Wolfram S. Cellular automata as models of complexity. Nature. International Weekly Journal of Science, 311:5985 (1984), 419- 424.

[8] Wolfram S. Tables of cellular automata properties. In "Theory and applications of cellular automata (included selected papers 19831986)"[Wolfram S. (Ed.)]. Advanced Series on Complex Systems. World Scientific Publishing, pp. 485-557, 1986.

[9] Martin O., Odlyzko A.M., Wolfram S. Algebraic Properties of Cellular Automata. Communications in Mathematical Physics, 93:2 (1984), 219-258.

[10] Pickard N.H. Wolfram S. Two-dimensional cellular automata. Journal of Statistics Physics, 38:5/6 (1985), 901-946.

[11] Biham O., Middleton A.A., Levine D. Self-organization and a dynamical transition in traffic flow models. Phys. Rev. A. American Physical Society,. 46:10 (1992), R6124-R6127.

[12] Belitzky V., Ferrary P.A. Invariant measures and convergence properties for cellular automation 184 and related processes. Journal of Statistical Physics, 118:3 (2005), 589-623.

[13] Blank M.L. Exact analysis of dynamical systems arising in models of traffic flow. Russian Mathematical Surveys, 2000, 55:3, 562-563.

[14] Gray L., Griffeath D. The ergodic theory of traffic jams, J. Stat. Phys., 3:4 (2001), 413-452.

[15] Buslaev A.P., Gasnikov A.V. , Yashina M.V. Selected Mathematical Problems of Traffic Flow Theory. International Journal of Computer Mathematics. 89: 3 (2012), 409-432.

[16] Kozlov V. V., Buslaev A. P. , Tatashev. A. G. A Dynamical Communication System on a Network. Journal of Computational and Applied Mathematics, 275 (2015), 257-261.

[17] D'Souza R.M. Coexisting phases and lattice dependence of a cellular automaton model for traffic flow. Phys. Rev. E., 71:6 (2005), 066112

[18] Angel, Omer; Holroyd, Alexander E.; Martin, James B. (12 Augus 2005). "The Jammed Phase of the Biham - Middleton - Levine Traffic Model". Electronic Communications in Probability. 10, 167 - 178.

[19] Austin T., Benjamini I. For what number of cars must self organization occur in the Biham - Middleton - Levine traffic model from any possible starting configuration/ (2006). arXiv: math/0607759.

[20] Kozlov V.V., Buslaev A.P., Tatashev A.G. Monotonic random walks and clusters flows on networks. Models and applications, Lambert Academician Publishing, Saarbrucken, Germany, 2013, no. 78724.

[21] Buslaev A.P., Tatashev A.G. Exact results for discrete dynamical systems on a pair of contours. Mathematical Methods in the Applied Sciences. (To appear)

[22] Kozlov V.V., Buslaev A.P., Tatashev A.G. On real-valued oscillations of a bipendulum. Applied Mathematics Letters, 2015, 46, 44 49

[23] Buslaev A.P., Tatashev A.G. On dynamical systems for transport logistic and communications. J. Math. Res., 2016, 8:4, 195 - 210.

[24] Buslaev A.P., Tatashev A.G. Bernoulli algebra on common fractions and generalized oscillations. J. Math. Res., 8:3 (2016), 82 - 95.

[25] Buslaev A.P., Yashina M. V. On holonomic mathematical F-pendulum. Mathematical Methods in the Applied Sciences,39:16 (2016), 4820 - 4828 